\title{
Relación entre el Movement change in karate position Test con el rendimiento neuromuscular en atletas de karate: Un estudio piloto \\ Relationship between Movement change in karate position Test and neuromuscular performance in karate athletes: A pilot study \\ *, **Tomas Herrera-Valenzuela, ${ }^{* *}$ Gricelle Miccono-González, ${ }^{* *}$ Margaret Fazekas-Molina, ${ }^{* *}$ Gonzalo Astorga-Rojas, ***Pablo Valdés-Badilla, ${ }^{* * * *}$ Alex Ojeda-Aravena, $* * * * *$ Emerson Franchini

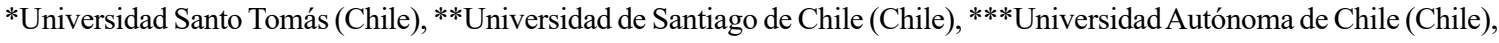

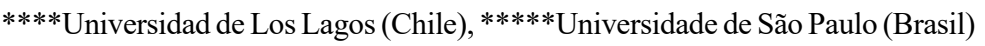

Resumen. Antecedentes: El karate es un deporte intervalado de alta intensidad con características aeróbicas y anaeróbicas. La velocidad y la fuerza explosiva son habilidades físicas determinantes para los atletas de karate durante el combate. Problema y objetivo: El principal objetivo de este estudio fue examinar la relación del Movement Change in Karate Position Test «MCKPT» con el rendimiento neuromuscular medido a través de pruebas de salto en atletas de karate categoría junior. Métodos: Una muestra de 10 atletas de karate, distribuidos en 4 hombres (edad: $17.3 \pm 2.1$ años; masa corporal: $69.0 \pm 15.8 \mathrm{~kg}$; talla: $1.74 \pm 0.04 \mathrm{~m}$ ) y 6 mujeres (edad: $17.3 \pm 1.6$ años; masa corporal: $57.9 \pm 2.5 \mathrm{~kg}$; talla: $1.59 \pm 0.04 \mathrm{~m}$ ), pertenecientes a la selección chilena categoría junior fue evaluada. Resultados: Observamos una correlación entre el MCKPT con el salto largo bilateral $(\mathrm{r}=-.68, p=.03)$, squat jump $(\mathrm{r}=-.65, p=.04)$, el salto contramovimiento $(\mathrm{r}=$ $-.70, p=.02)$, y salto contramovimiento con brazos $(\mathrm{r}=-.68, p=.03)$. Conclusiones: La prueba MCKPT se relaciona significativamente con el rendimiento neuromuscular en atletas de karate.

Palabras clave: Deportes, deporte de combate, agilidad, rendimiento de salto, karate.

\begin{abstract}
Background: Karate is a high-intensity interval sport with aerobic and anaerobic characteristics. Velocity and explosive strength are crucial physical abilities for karate athletes during the combat. Problem and Aim: The main objective of this study was to examine the relationship of the Movement Change in Karate Position Test «MCKPT» with the neuromuscular performance measured through jump tests in junior karate athletes category Methods: A team of 10 karate athletes, distributed in 4 men (age: $17.3 \pm 2.1$ years; body mass: $69.0 \pm 15.8 \mathrm{~kg}$; height: $1.74 \pm 0.04 \mathrm{~m}$ ) and 6 women (age: $17.3 \pm 1.6$ years; body mass: $57.9 \pm 2.5 \mathrm{~kg}$; height: $1.59 \pm 0.04 \mathrm{~m}$ ), belonging to the Chilean national junior team was evaluated. Results: We observed a correlation between the MCKPT with the bilateral long jump $(\mathrm{r}=-.68, \mathrm{p}=.03)$, squat jump $(\mathrm{r}=-.65, \mathrm{p}=.04)$, countermovement jump $(\mathrm{r}=-.70, \mathrm{p}=.02)$, and countermovement jump with $\operatorname{arms}(\mathrm{r}=-.68, \mathrm{p}=.03)$. Conclusions: MCKPT is significantly related to neuromuscular performance in karate athletes.
\end{abstract}

Key words: Sports, combat sports, agility, jump performance, karate.

\section{Introducción}

El karate es un deporte intermitente y de alta intensidad con características aeróbicas y anaeróbicas (Beneke, Beyer, Jachner, Erasmus, \& Hutler, 2004; Doria et al., 2009). El rendimiento aeróbico participa en la recuperación entre movimientos y entre combates, mientras que el rendimiento anaeróbico es fundamental en la ejecución de movimientos explosivos que permiten marcar puntos (Chaabène, Hachana, Franchini, Mkaouer, \& Chamari, 2012).

La velocidad y la fuerza explosiva son capacidades físicas determinantes para los atletas de karate durante el combate (Chaabène et al., 2012; Loturco, Artioli, Kobal, Gil, \& Franchini, 2014). Adicionalmente los atletas de karate necesitan acelerar de manera lineal y en diferentes direcciones para atacar y contratacar, situación similar a otros deportes de combate como el taekwondo (Ojeda-Aravena, AzócarGallardo, Hérnandez-Mosqueira, \& Herrera-Valenzuela, 2021). Por lo tanto, la fuerza explosiva y la agilidad son necesarias para realización de movimientos rápidos y explosivos durante la competencia de karate (Chaabène et al., 2012).

Sin embargo, habitualmente, estas variables son medidas a través de evaluaciones generales con movimientos cíclicos que no representan las características del deporte

Fecha recepción: 23-06-20. Fecha de aceptación: 13-08-20

Tomas Herrera-Valenzuela

tomas.herrera@usach.cl
(Chaabene et al., 2018). Por ejemplo, el rendimiento neuromuscular es evaluado a través de pruebas de salto, como el salto contramovimiento (CMJ) y squat jump (SJ) (Chaabène et al., 2012). Por otro lado, recientemente se han desarrollado pruebas específicas para deportes de combate relacionadas con la agilidad (Chaabene et al., 2018), en particular, para atletas de karate se ha propuesto la prueba denominada «Movement change in karate position» (MCKPT) (Chaabene et al., 2018; Sertic, Vidranski, \& Segedi, 2011), sin embargo, su relación con el rendimiento de salto no ha sido examinada.

Aunque en otros deportes de combate como taekwondo (Chaabene et al., 2018; Ojeda-Aravena et al., 2021) y esgrima (Turner et al., 2016) se ha encontrado relación entre el rendimiento del salto y la agilidad medida de forma específica, hasta donde los autores sabemos, esto no ha sido investigado en atletas de karate junior. Conocer la relación entre el rendimiento en el salto y la agilidad específica es un aspecto relevante al considerar que el rendimiento en el salto es significativamente diferente entre atletas de karate junior de distinto nivel competitivo (Ravier, Grappe, \& Rouillon, 2004). Por lo tanto, el objetivo de esta investigación fue analizar la relación del MKUKS con el rendimiento neuromuscular medido a través de pruebas de salto en atletas elite de karate categoría junior. Se hipotetiza que el MKUKS es una prueba que se relaciona con el rendimiento de salto, ya que atletas con más potencia pueden acelerar y desacelerar de forma más intensa en una prueba de agilidad. 


\section{Material y métodos}

\section{Participantes}

Estudio descriptivo correlacional, con una muestra por conveniencia de 10 atletas de karate, distribuidos en 4 hombres (edad:17.3 \pm 2.1 años; masa corporal: $69.0 \pm 15.8 \mathrm{~kg}$; talla: $1.74 \pm 0.04 \mathrm{~m}$ ) y 6 mujeres (edad: $17.3 \pm 1.6$ años; masa corporal: $57.9 \pm 2.5 \mathrm{~kg}$; talla: $1.59 \pm 0.04 \mathrm{~m}$ ), pertenecientes a la selección chilena de la categoría junior.

Los criterios de inclusión fueron: i) al menos dos años de experiencia en entrenamiento de karate; ii) entrenar al menos cinco veces por semana; iii) estar en proceso de preparación para competiciones o torneos organizados por la Federación Deportiva Nacional de Karate de Chile, organización reconocida por la World Karate Federation (WKF), iv) tener al menos dos meses de entrenamiento ininterrumpido. Mientras que los criterios de exclusión fueron: i) tener una lesión o trastorno físico que los inhabilitaría de la práctica deportiva; ii) consumo de algún suplemento nutricional o medicamento que modifique el rendimiento; iii) encontrarse en proceso de disminución de peso corporal.

Todos los participantes fueron informados verbalmente y por escrito sobre el propósito, los métodos y los medios del estudio. Los padres de los participantes firmaron un consentimiento que autoriza el uso de la información con fines científicos, mientras que a los atletas también se les solicitó firmar un asentimiento. El protocolo de investigación fue revisado y aprobado por el Comité de Ética Científica y se desarrolló siguiendo la Declaración de Helsinki.

\section{Procedimientos y medidas}

Las medidas se llevaron a cabo en el Centro de Entrenamiento Olímpico de Chile. durante enero de 2019. Los participantes se presentaron en el laboratorio para la medición de la prueba específica de agilidad (MKUKS), el salto largo bilateral (SLB), el SJ, el CMJ, y el CMJ con utilización de brazos (CMJ-brazos). Los participantes llegaron al laboratorio a las 8:00 a.m. Antes de la sesión de medición a los participantes se les pidió abstenerse de hacer ejercicio más allá de lo requerido para el estudio y mantener su dieta normal.

Movement change in karate position (MKUKS)

Para evaluar la agilidad de manera específica se utilizó la prueba MKUKS. Esta prueba estuvo compuesta de dos líneas paralelas en el suelo de 1 metro de largo y 2 metros de distancia entre ellas. Los participantes se pararon en posición de combate frente a la primera línea. Después de la señal de inicio, los participantes se movieron lo más rápido posible en hop-step (sori ashi), sin cruzar las piernas, moviéndose hacia la segunda línea. Cuando el atleta pisó con el pie delantero la segunda línea, se detuvo girando 180 grados y volviendo a la primera línea, para tocar nuevamente con el pie adelantado la primera línea. El movimiento se repitió seis

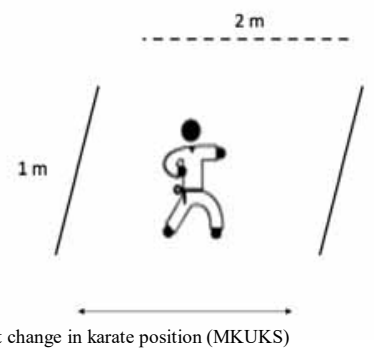

Figura 1. Movement change in karate position (MKUKS) veces y se registró la duración total de la prueba (Sertic et al., 2011). La prueba fue filmada a través de un iPhone 7 ubicado en un trípode, posteriormente el video fue analizado a través del software Kinovea para el registro del tiempo de ejecución. Cada participante ejecutó la prueba tres veces con 2 minutos de recuperación entre intentos. Se registró el mejor rendimiento para el análisis (Figura 1).

Para evaluar la fuerza explosiva concéntrica de manera indirecta se utilizó el SJ a través de la altura máxima alcanzada (cm) del salto vertical. La prueba SJ se realizó utilizando una plataforma de contacto electrónica (Ergojump; Globus, Codogne, Italia) con una precisión de $0.01 \mathrm{~m}$. Durante el SJ se les indicó a los participantes que apoyaran las manos sobre las caderas, los pies bien separados, adoptaran una posición de rodillas flexionadas (aproximadamente 90 grados) durante 3 segundos, y luego realizaran un salto vertical a su máximo esfuerzo. Todos los participantes recibieron instrucciones para aterrizar en posición vertical y flexionar las rodillas después del aterrizaje (Ramírez-Campillo, Andrade, \& Izquierdo, 2013). Se completaron tres intentos, con un minuto de recuperación entre intentos (Moran et al., 2017), se consideró el mejor rendimiento para los análisis posteriores.

\section{Salto contramovimiento (CMJ)}

Para evaluar el ciclo de estiramiento-acortamiento lento se utilizó el CMJ considerando la altura máxima alcanzada (cm) del salto. Se utilizó una plataforma de contacto electrónica (Ergojump; Globus, Codogne, Italia) con una precisión de $0.01 \mathrm{~m}$. Durante el CMJ se les indicó a los participantes que apoyaran las manos sobre las caderas y los pies bien separados. Los participantes realizaron un movimiento hacia abajo (no se impuso ninguna restricción sobre el ángulo de rodillas alcanzado) seguido de un salto vertical de esfuerzo máximo. Todos los participantes recibieron instrucciones de aterrizar en posición vertical y flexionar las rodillas después del aterrizaje (Ramírez-Campillo et al., 2013). Se completaron tres intentos, con un minuto de recuperación entre intentos (Moran et al., 2017), se consideró el mejor rendimiento para los análisis posteriores.

\section{Salto contramovimiento con brazos (CMJ-brazos)}

Se utilizó CMJ-brazos considerando la altura máxima alcanzada $(\mathrm{cm})$ del salto vertical. La prueba CMJ-brazos se realizó utilizando una plataforma de contacto electrónica (Ergojump; Globus, Codogne, Italia) con una precisión de $0.01 \mathrm{~m}$. Durante el CMJ-brazos se les indicó a los participantes que tuvieran las manos libres para moverse y los pies bien separados. Los participantes realizaron un movimiento hacia abajo (no se impuso ninguna restricción sobre el ángulo de rodilla alcanzado) seguido de un salto vertical de esfuerzo máximo usando los brazos libremente. Al igual que en el CMJ, todos los participantes recibieron instrucciones de aterrizar en posición vertical y flexionar las rodillas después del aterrizaje (Ramírez-Campillo et al., 2013). Se completaron tres intentos, con un minuto de recuperación entre intentos (Moran et al., 2017), se consideró el mejor rendimiento para los análisis posteriores.

Salto largo bilateral (SLB)

Para valuar la fuerza explosiva horizontal se utilizó el sal- 
to largo bilateral siguiendo recomendaciones previas (Loturco et al., 2018). Brevemente se solicitó a los participantes que se pararan con los dedos de los pies alineados a un marcador negro ubicado en el suelo. Los participantes recibieron instrucciones de realizar un salto horizontal después de ponerse en cuclillas a una profundidad autoseleccionada con el objetivo de saltar lo más adelante posible. Para esta prueba se permitió un balanceo de los brazos debido a la mayor dificultad. Se realizaron dos intentos con un descanso de 3 minutos entre intentos y el intento de mejor rendimiento se utilizó para el análisis estadístico posterior.

\section{Análisis Estadístico}

El programa estadístico SPSS $®$ versión 26.0, fue utilizado para los análisis. Las medianas, percentiles 25 y 75, e intervalo de confianza al $95 \%$ fueron obtenidas para todas las variables. Además, fue contrastada la normalidad de las variables utilizando la prueba Shapiro-Wilk. Para establecer la relación entre las variables se utilizó la prueba de correlación de Pearson. Para todos los casos se estableció un nivel de significancia de $\mathrm{p}<0.05$.

\section{Resultados}

La Tabla 1 presenta el rendimiento para las pruebas de salto (SLB, SJ, CMJ, CMJ-brazos) y la prueba de agilidad específica (MKUKS). Mientras que la Figura 2 presenta la correlación entre MKUKS con SLB, SJ, CMJ y CMJ-brazos.

\begin{tabular}{|c|c|c|c|c|c|}
\hline Grupo & SLB $(m)$ & SJ $(\mathrm{cm})$ & CMJ $(\mathrm{cm})$ & CMJ-brazos $(\mathrm{cm})$ & MKUKS (s) \\
\hline \multicolumn{6}{|l|}{ Hombres } \\
\hline Media $\pm \mathrm{DE}$ & $2.26 \pm 0.19$ & $34 \pm 6$ & $38 \pm 7$ & $43 \pm 8$ & $9.13 \pm 1.19$ \\
\hline IC $95 \%$ & $1.96-2.56$ & $25-43$ & $28-49$ & $30-56$ & 7.23-11.02 \\
\hline \multicolumn{6}{|l|}{ Mujeres } \\
\hline Media $\pm \mathrm{DE}$ & $1.84 \pm 0.19$ & $24 \pm 4$ & $25 \pm 5$ & $30 \pm 4$ & $9.67 \pm 0.95$ \\
\hline IC $95 \%$ & $1.64-2.04$ & $20-27$ & $19-30$ & $26-33$ & $8.67-10.67$ \\
\hline \multicolumn{6}{|l|}{ Total } \\
\hline Media $\pm D E$ & $2.01 \pm 0.28$ & $28 \pm 7$ & $30 \pm 9$ & $35 \pm 9$ & $9.45 \pm 1.03$ \\
\hline IC $95 \%$ & $1.81-2.21$ & $23-33$ & $24-36$ & $29-41$ & $8.71-10.19$ \\
\hline
\end{tabular}
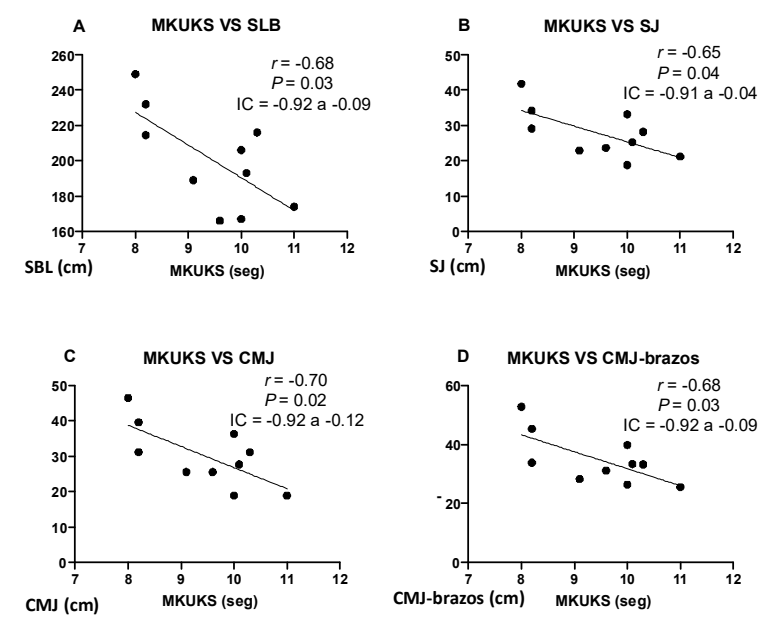

Figura 2. Correlación entre el rendimiento de la prueba de agilidad específica (MUMKS) con el rendimiento en las pruebas de salto en atletas elite de karate categoría junior. Panel A: correlación entre "Movement change in karate position test" versus "Salto largo bilateral"; Panel B: correlación entre "Movement change in karate position test" versus "Squa jump"; Panel C: correlación entre "Movement change in karate position test" versus "Salto contramovimiento"; Panel D: correlación entre "Movement change in karate position test" versus "Countermovement jump con brazos"; r: r r de Pearson; P: valor de significancia; IC intervalo de confianza $95 \%$.

\section{Discusión}

El propósito de este estudio fue examinar la relación del MKUKS con el rendimiento neuromuscular medido a través de pruebas de salto en atletas elite de karate categoría junior. El principal hallazgo fue la relación significativa existente entre el MKUKS con el rendimiento del SLB, el SJ, el CMJ y el CMJ-brazos, por lo tanto, los entrenadores podrían utilizar el MKUKS como un indicador del rendimiento neuromuscular en atletas de karate.

Los atletas de este estudio obtuvieron un rendimiento de $9.13 \pm 1.19$ s para completar el MKUKS, valores algo más elevados que los reportados en un estudio previo (Sertic et al., 2011), donde se informaron valores entre $7.21 \pm 1.93 \mathrm{~s}, \mathrm{y}$ $6.84 \pm 1.88 \mathrm{~s}$ durante diferentes intentos en una muestra de 65 karatecas de $12 \pm 2.5$ años de edad. La agilidad y la fuerza explosiva son elementos determinantes en el rendimiento de aletas de karate (Chaabène et al., 2012). En este sentido, nuestros resultados muestran una relación entre el MKUKS y las pruebas de salto, específicamente, con SLB, SJ, CMJ y CMJbrazos, lo cual concuerda con la relación existente entre la agilidad medida a través de pruebas con carrera y la potencia (Sheppard \& Young, 2006), hecho relevante, al considerar que el rendimiento en el salto es significativamente diferente entre atletas de karate junior de distinto nivel competitivo (Ravier, Grappe, \& Rouillon, 2004).

Además, estos datos son consistentes con estudios previos realizados en otros deportes de combate que han analizado la relación entre las características de la fuerza explosiva y el rendimiento de la agilidad de manera específica. Por ejemplo, a través de la prueba específica de agilidad en taekwondo (TSAT) en atletas de élite se reportó una relación significativa e inversa con $\mathrm{SJ}(\mathrm{r}=-0.62 ; \mathrm{p}<0.001)$, $\mathrm{SLB}(\mathrm{r}=$ $0.60 ; \mathrm{p}<0.001)$ y CMJ $(\mathrm{r}=-0.43 ; \mathrm{p}=0.02)$ (Chaabene et al., 2018). Asimismo, en atletas de taekwondo de nivel nacional se reportaron asociaciones inversas grandes en $\mathrm{SJ}(\mathrm{r}=-0.63$; $\mathrm{p}<0.01), \mathrm{CMJ}(\mathrm{r}=-0.56 ; \mathrm{p}<0.03)$ y TSAT (Ojeda-Aravena et al., 2021). A su vez, mediante el coeficiente de determinación (R2), los autores señalaron que tanto las características concéntricas y el ciclo estiramiento acortamiento de la fuerza explosiva podrían haber explicado el rendimiento obtenido en un $40 \%$ y $32 \%$, respectivamente (Ojeda-Aravena et al., 2021). Por otra parte, un estudio con atletas de esgrima, que utilizó la prueba de 4-2-2-4-m, reportó una asociación inversa grande con el SLB $(r=-0.65)$ y moderada con CMJ $(r=-0.49)$, mientras que mediante R2 estimaron que el SLB representó un $43 \%$ en la variabilidad de los resultados obtenidos (Turner et al., 2016).

Dentro de las principales fortalezas del estudio, se encuentra; i) la utilización de una prueba específica de karate, situación relevante al considerar la escaza información al respecto; ii) los participantes del estudio pertenecen a un equipo nacional de la categoría junior. Mientras que la principal limitación de este estudio fue el tamaño de la muestra.

En conclusión, el MKUKS se relaciona significativamente con el rendimiento neuromuscular en atletas elite de karate categoría junior. Lo interesante del hallazgo radica en la facilidad para ejecutar el MKUKS, que con poca implementación, los entrenadores podrían controlar las modificaciones del rendimiento neuromuscular en sus deportistas. 


\section{Referencias}

Beneke, R., Beyer, T., Jachner, C., Erasmus, J., \& Hutler, M. (2004). Energetics of karate kumite. European Journal of Applied Physiology, 92(4-5), 518-523. https://doi.org/ 10.1007/s00421-004-1073-x

Chaabène, H., Hachana, Y., Franchini, E., Mkaouer, B., \& Chamari, K. (2012). Physical and Physiological Profile of Elite Karate Athletes. Sports Medicine, 42(10), 829-843. https://doi.org/10.2165/11633050-000000000-00000

Chaabene, H, Negra, Y., Bouguezzi, R., Capranica, L., Franchini, E., Prieske, O., ... Granacher, U. (2018). Tests for the Assessment of Sport-Specific Performance in Olympic Combat Sports: A Systematic Review With Practical Recommendations. Frontiers in Physiology, 9. https://doi.org/10.3389/FPHYS.2018.00386

Chaabene, Helmi, Negra, Y., Capranica, L., Bouguezzi, R., Hachana, Y., Rouahi, M. A., \& Mkaouer, B. (2018). Validity and Reliability of a New Test of Planned Agility in Elite Taekwondo Athletes. Journal of Strength and Conditioning Research, 32(9), 2542-2547. https:// doi.org/10.1519/JSC.0000000000002325

Doria, C., Veicsteinas, A., Limonta, E., Maggioni, M. A., Aschieri, P., Eusebi, F., ... Pietrangelo, T. (2009). Energetics of karate (kata and kumite techniques) in toplevel athletes. European Journal of Applied Physiology, 107(5), 603-610. https://doi.org/10.1007/s00421-009-1154

Loturco, I., Artioli, G. G., Kobal, R., Gil, S., \& Franchini, E. (2014). Predicting punching acceleration from selected strength and power variables in elite karate athletes: a multiple regression analysis. The Journal of Strength \& Conditioning Research, 28(7), 1826-1832.

Loturco, I., Nimphius, S., Kobal, R., Bottino, A., Zanetti, V., Pereira, L. A., \& Jeffreys, I. (2018). Change-of direction deficit in elite young soccer players. German Journal of
Exercise and Sport Research, 48(2), 228-234. https:// doi.org/10.1007/s12662-018-0502-7

Moran, J., Sandercock, G., Ramírez-Campillo, R., Todd, O., Collison, J., \& Parry, D. (2017). Maturation-Related Effect of Low-Dose Plyometric Training on Performance in Youth Hockey Players. Pediatric Exercise Science, 29(2). https://doi.org/10.1123/PES.2016-0151

Ojeda-Aravena, A. P., Azócar-Gallardo, J., HérnandezMosqueira, C., \& Herrera-Valenzuela, T. (2021). Relación entre la prueba de agilidad específica en taekwondo (tsat), la fuerza explosiva y la velocidad líneal en 5-m atletas de taekwondo de ambos sexos. RETOS, Nuevas Tendencias En Educación Física, Deportes y Recreación, 39, 84-89.

Ramírez-Campillo, R., Andrade, D., \& Izquierdo, M. (2013). Effects of plyometric training volume and training surface on explosive strength. The Journal of Strength \& Conditioning Research, 27(10), 2714-2722.

Ravier, G., Grappe, F., \& Rouillon, J. D. (2004). Application of force-velocity cycle ergometer test and vertical jump tests in the functional assessment of karate competitor. Journal of Sports Medicine and Physical Fitness, 44(4), 349.

Sertic, H., Vidranski, T., \& Segedi, I. (2011). Construction and Validation of Measurement Tools for the Evaluation of Specific Agility in Karate. Ido Movement for Culture. Journal of Martial Arts Anthropology, 11(1), 37-41.

Sheppard, J., \& Young, W. (2006). Agility Literature Review: Classifications, Training and Testing. Journal of Sports Sciences, 24(9). https://doi.org/10.1080/ 02640410500457109

Turner, A., Bishop, C., Chavda, S., Edwards, M., Brazier, J., \& Kilduff, L. P. (2016). Physical Characteristics Underpinning Lunging and Change of Direction Speed in Fencing. Journal of Strength and Conditioning Research, 30(8), 2235-2241.https://doi.org/10.1519/JSC.0000000000001320

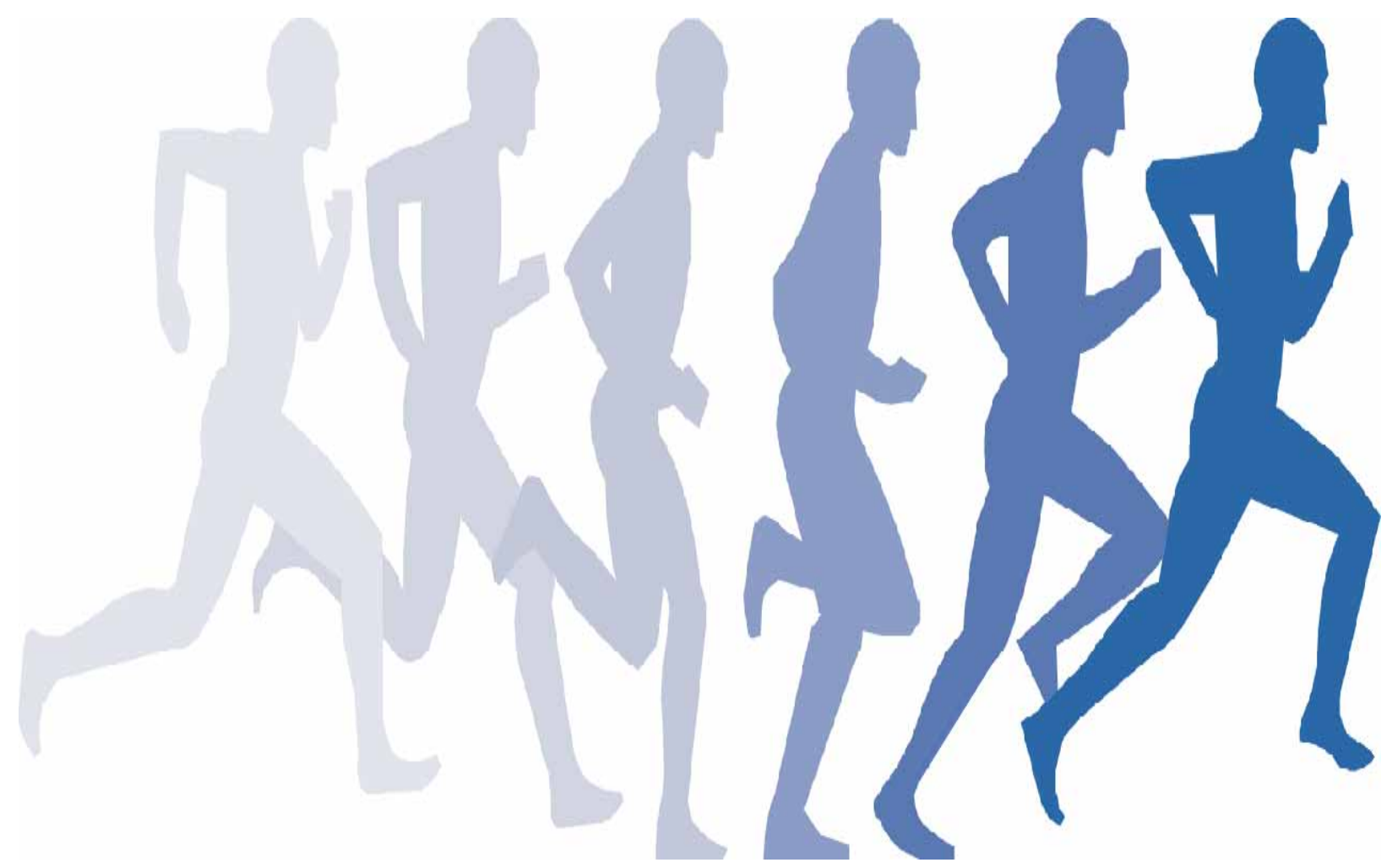

